\title{
Critical Algorithm for Graph and Image Compression and Transmission Research
}

\author{
Jun Tang \\ Department of Communication Engineering, Xiamen University of Technology, \\ Xiamen, Fujian 361024, China \\ E-mail:xmtangjun@126.com
}

\begin{abstract}
This article is dedicated to the purpose of introducing the simplification of the triangular-network's multi-resolution, as well as the concept of grid, trigonometry, topographic goading, triangular-sector, etc. In addition, this article proposes a new algorithm that combines the layer-segmentation of the multi-resolution model and the binary space partitioning $(B S P)$ tree, in which the layer-segmentation of the triangular belt is capable of achieving a better result in terms of dissolving the model, therefore creating the foundation of the BSP tree's structural setup. The purpose of finding BSP tree, on the other hand, is to identify sensitive data from the non-sensitive one, so that whatever data that is contacted with the viewpoint can be fully transferred and displayed. Speaking of the designing part of the database, this algorithm combines the latest techniques such as the XML.ADO.NET, and thereby capable of designing transfer plans accordingly.
\end{abstract}

Keywords: Graph and image processing, binary space partitioning, layersegmentation, multi-resolution model.

\section{Introduction}

The compression of geometric model is divided into single-resolution compression and multi-resolution compression. On the compression of singleresolution model, such as Deer algorithm, real-time compression algorithm on connection relationship of triangular mesh put forward by Gumhold, Taubin and Rossignac's compression algorithm based on topological surgery, are some typical algorithms [1]. Multi-resolution model can be segmented into discrete multiresolution model and continuous multi-resolution model. Discrete multi-resolution model simplifies the complex triangle mesh to get more triangular mesh models with different levels of detail through simplified algorithm, however, with a compact representing method for model, continuous multi-resolution model can generate different resolution models with any number. Based on the two different models, object-oriented compression of multi-resolution model can be divided into object-oriented compression of discrete multi-resolution model and object-oriented compression of continuous multi-resolutionn model [2].

To any network, Hoppe has put forward the representation of progressive meshes for surface geometric modeling, which simplifies the network by optimizing the energy function. Progressive Mesh can provide any connection levels of detail (LOD) grid. This tiered representation can be used in multi-resolution editing and progressive transmission. But rough approximation may have a serious artificial trace, and this kind of data format is difficult to be compressed for the amount of data for progressive transmission is higher than that of original grid data. 
Progressive forest split compression was put forward by G. Taubin in 1998. The algorithm has adopted more complex and detailed operations than progressive mesh like Hoppe, which can more effectively compress and encode.

The characteristic of this scheme is that it had employed an adaptive elaboration method which allows smooth transition between continuous and different levels of details for elaboration model when storage and transit triangular mesh which stores in a progressive and deep compressed method [3]. The forest part can be regarded as a set with a series of sides split operations but not as a series of independent operations. Multi-resolution in perfect forward secrecy (PFS) format is made up of an initial low-resolution LOD and a variety of forest part operations. Among them, the lowest-resolution model is expressed with topological operation method.

In some previous methods, many researchers used Delaunay which has constraint to delete left hole. The advantage of this method is that it can avoid the narrow triangle as much as possible for that the triangulated triangles can have a good founder rate. But in the Delaunay, it fails to consider the errors and also it is difficult to consider the errors between simplified geometric model and nonsimplified geometric model, so the quality of simplification is not so good [4].

\section{The Concept of Triangular Grid}

Before describing the geometry compression algorithm in this section, the representing method and some related base concepts will be introduced first in detail. Although triangular mesh can be described by using graphs in essence, but the following representing method named "vertex-triangle" which is more intuitive is adopted.

A triangle grid can be expressed as a binary group. They are respectively a set of vertices and triangles for triangular mesh $M$. Among them, each element of $t$ can be expressed as cyclic triple made up of elements in set $V$ of vertices in the set $T$ of the triangle [5].

$$
\begin{gathered}
M=(V, T) \\
V=\left\{v_{i} \mid i=0,1,2, \cdots, N-1\right\} \\
T=\{t \mid i=0,1,2, \cdots, M-1\} \\
t=\left(v_{0}, v_{1}, v_{2}\right)
\end{gathered}
$$

In this cyclic triple group, the arrangement of vertical elements obeys the traditional right-hand rule, is called the set of vertices for the triangle. Supposing is ordered vertices, if they are two vertices of the triangle at the same time, then we'll call the ordered vertices as a side. The set of all the sides is denoted as $E$ on the triangular mesh $M$.

\section{Algorithm Research}

\subsection{The Coding and Multi-layer Model of the Bi-orthogonal Wavelet}

According to the wavelet designed, which holds the property of linear-phase and symmetrised to the use of the segmentation of the original grid, $M_{N}$. After the switch, mark the current grid as $M_{N-1}$, and call all definitive entity as that of the $M_{N}$, note as $D_{N}$. The previous grid is hereby noted as the sum of $M_{N-1}$ and $D_{N}$, with the formula:

$$
M_{N}=M_{N-1} \oplus D_{N}
$$


By separating the original grid, we are able to decompose the ordinal grid $M_{N}$ as the sum of one base grid $M_{0}$ and multi-layer definitive $D_{N}$.

The expression of the multi-resolution contains two parts: the first part is the base grid $M_{0}$, it is the simplest form of algebra, for the base grid is normally extremely small, therefore, one may even proceed without compressing it [6]. The second part is definitive $D_{l}$, it is considered as an important factor of the multiresolution algebraic compression process to code and compress definitive $D_{l}$.

The wavelet's altering range will then threshold the coefficient, filtering out those that are smaller than the threshold. In order to minimize the wavelet's officious depletion after the threshold, meanwhile approximately $94 \%$ of the wavelet coefficient is zero, the threshold is thusly decided by the actual energy situation after the exchange, the self-fitting method, in this case, is capable of making nearly $94 \%$ of the entire coefficient population to zero [7]. Based on the perspective of minimum error before and after the compression, this can greatly reduce the time that is needed for the system to process the data.

After altering the wavelet and qualifying the threshold, the grid definitive, ranging from $D_{I}$ to $D_{N}$, suits perfectly for run-length encoding [8]. For data that lies within the gird $M_{0}$, the entropy-encoding method - the Huffman encoding, should be considered as the very best option. Doing so allows the combination of the two entropy encoding, the base grid and grid definitive perfectly to proceed swimmingly, so that the very best compression result is thusly achieved.

\subsection{Topographic Coding}

In normal sense, the polygonal grid can be considered as an entity that consists of polygons with multiple cited properties, together, these polygon entities merge as a three-dimensional surface with all the attached original properties, which includes the climax coordinates, the colour, the vectoring factor and the texturing coordinates. The overall information of the polygonal grid can be viewed as three parts:

(1) The topographic information is designated to describe the interaction between climax and surface;

(2) The trigonometric information is to describe the coordinates of each and every climax that were concluded within the polygonal grid;

(3) The properties such as the colour, and vectors of the climax. In order to solve the coding issue regarding the interaction between the grid's climax, one possible methodology is the dynamic coverage of one single climax cycling chain and one active slip that is located on it, thereafter commence the exploring and coding procedure towards the triangular grid.

When it comes to coding, the critical thinking will be to locate the triangle that is related to the current active slip in the first place, then begin the coding for triangles regarding its third climax [9]. When it comes to coding the terminals, one should stick to the Huffman coding, which is signified by providing data flow that has larger probability occurrence with shorter coding process, while favouring those with smaller probability with the opposite choice. It is in fact that under the circumstances where the probability is given, Huffman coding contains the least unnecessary information, the average length is the shortest. Hence, we conclude that Huffman coding is the very best solution.

\subsection{The Triangular Belt}

The triangular belt is being applied as a common methodology when involved into the field of topology. It includes steroid triangular belt, the zig-zag triangular belt, and the general triangular belt. These different belt can be described as a 
sequence that contains the coordinates of a climax. The sequence, nevertheless, can be transferred directly into the graphing tunnel. However, speaking of each triangle's input result, these triangles are not isolated from one another, the occurrence of the common climax will be expected as a popular phenomenon [10]. Therefore, the easiest way to do the topography is to repeatedly making the same coordinate input, which lags the graphic hardware's processing efficiency significantly. To solve this, one climax buffer device is needed. By applying the buffer, these common climax's coordinates will be stored elsewhere temporarily during its first application. It could thusly be retrieved directly from the buffer later on instead of making another input [11-13].

\subsection{The General Triangular Belt}

The general triangular belt is the most complicated belt theory proposed by Deering [14]. It is capable of compositing new triangle by the means of the zig-zag triangular belt, as well as that of the steroid triangular belt. Besides, it can also create triangles that shares no common border, climax, and the way that those points sequence. The price for possessing such capabilities is a 2-bit controlling digits has to be added in front of each and every one of the points [15-17]. When commencing Dearing's coding procedure, it is essential to quantify and compress climax's properties as the coordinates, colour, vector, texture, etc.

\subsection{The Pent-layer Segmentation}

In the case of data processing, all triangular belt methodologies aforementioned are required to maintain a huge body of data structure, which is of great disadvantage in terms of internet data transfer [18]. In order to get the issue resolved, one may lower the complicity and data quantity of the grid data transfer by redefining two triangular multi-layer structure, the $\mathrm{O}$ shape layer and the $\mathrm{U}$ shape layer (the $\mathrm{O}$ shape layer, in the case of need, can be segmented into the form of $U$ shape layer), to achieve the goal of algebraic simplification.

An example of the primitive grid's segmentation is shown below in Figure 1.

Figure 1(b) $\mathrm{O}$ shape layer, while Figure 1(a) the adjacent layer 2 is $\mathrm{U}$ shaped. Even though most of the layers are simple, the rest are complicated. Therefore, one has to degrade the complicate layers into those that are more simplified, and more easily to be processed by coding. Any layer form can be segmented into these two basic layer structure.

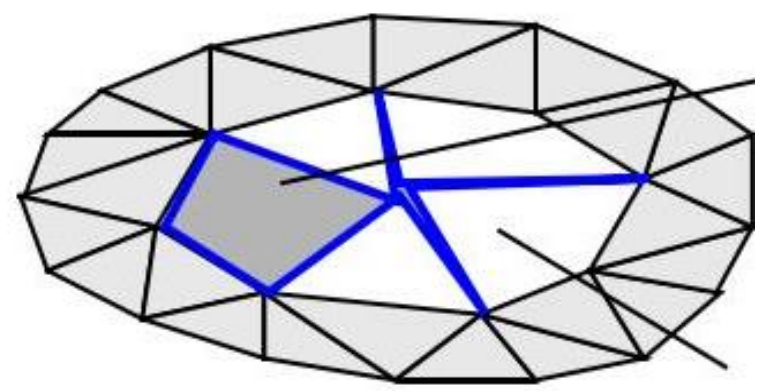

(a)

Figure 1 (a). U shape layer 


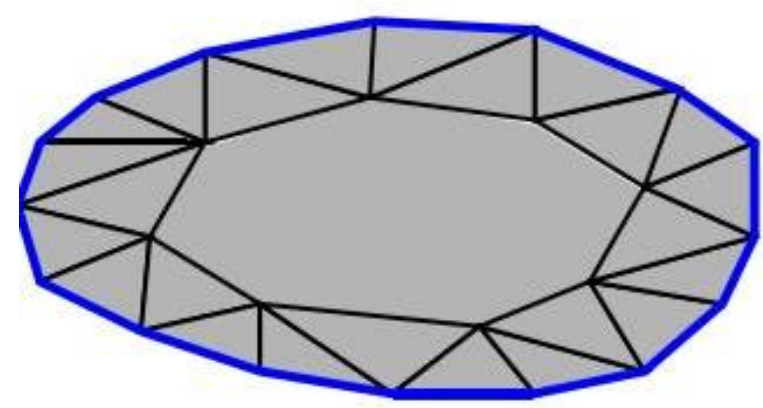

(b)

Figure 1 (b). O Shape Layer

\subsection{BSP Tree}

The BSP tree is a standard two-branch tree that are being used to sequence/search for the object that orients itself within the n dimensional space. The BSP tree, when applying into practice, represents the entire entity, in which every single branch terminal represents one reflexive space [19]. Every terminal conceives one "megaplane" as the cropping plane of the bisectional one, nevertheless, its two affiliated terminals represent two cropped inferior planes separately. In addition, each terminal can also include one or multiple algebraic objects [20]. The triangle of the grid data's incoming terminal sector generates based on the relative position between eyes and the current terminal's cropping plane. The terminals that lie among it contains layer information and that of the triangular-terminal one.

The backbone algorithm regarding the BSP generation:

1) Identify the relative position between the viewpoint and the current terminal cropping plane.

2) In the case that the viewpoint lies in front of the cropping plane, the process of identifying the resolution and distilling layer and triangular information based on the back side of the inferior space is prioritized [21-23].

3 ) In the case that the viewpoint lies behind the cropping plane, the process of identifying the resolution and distilling layer and triangular information based on the front side of the inferior space is prioritized.

4) In the case that the viewpoint is located within the cropping plane, one shall select any inferior space to process the layer information and triangular information. Through the generation of the two-branch tree and the distillation of triangular information visible layer, the quantity of transferable triangles is thusly simplified.

\subsection{Brief on the Visual-relevant Transfer Algorithm}

Speaking of the visual-relevant transfer algorithm, what comes in mind first is coding and the topographic information of the transfer model. What comes next is the expression of the multi-layer structure that is founded within various sectors of the algebraic along with visual sensitivity. Later on, based on the structure previously designed, the algebraic information is to be coded and transferred from the low layer to that of the high one. Referencing the coding/decoding algorithm description from Figure 2 and 3, by using the same method, the receiving end is also capable of setting up an identical layer structure automatically, meanwhile capable of rebuilding the algebraic model. Comparing to the transfer method 
designed by the spirit of this article, the difference is that this algorithm only requires layers from the visible area of the algebraic model, and the data of the triangular region.

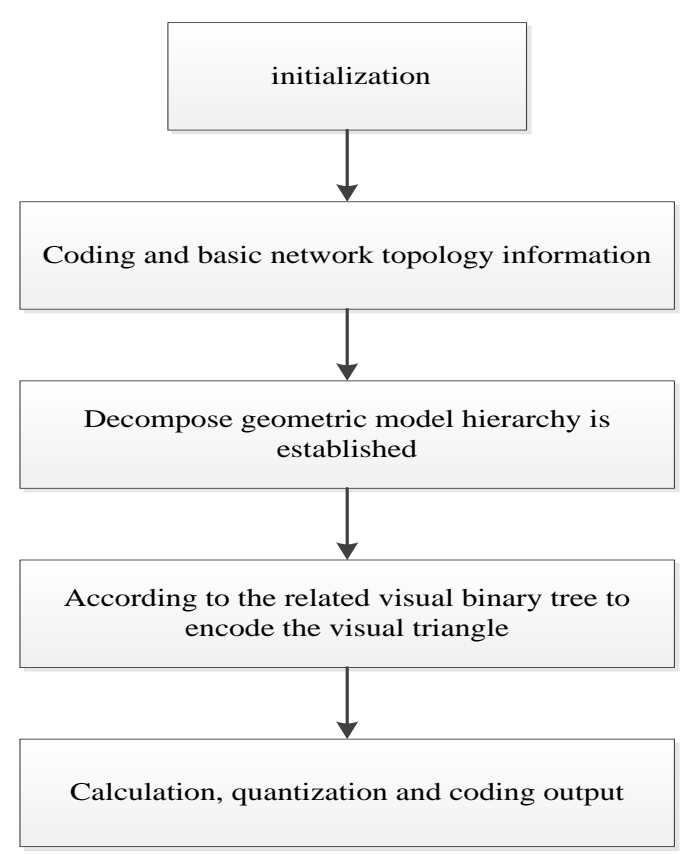

Figure 2. Code Description

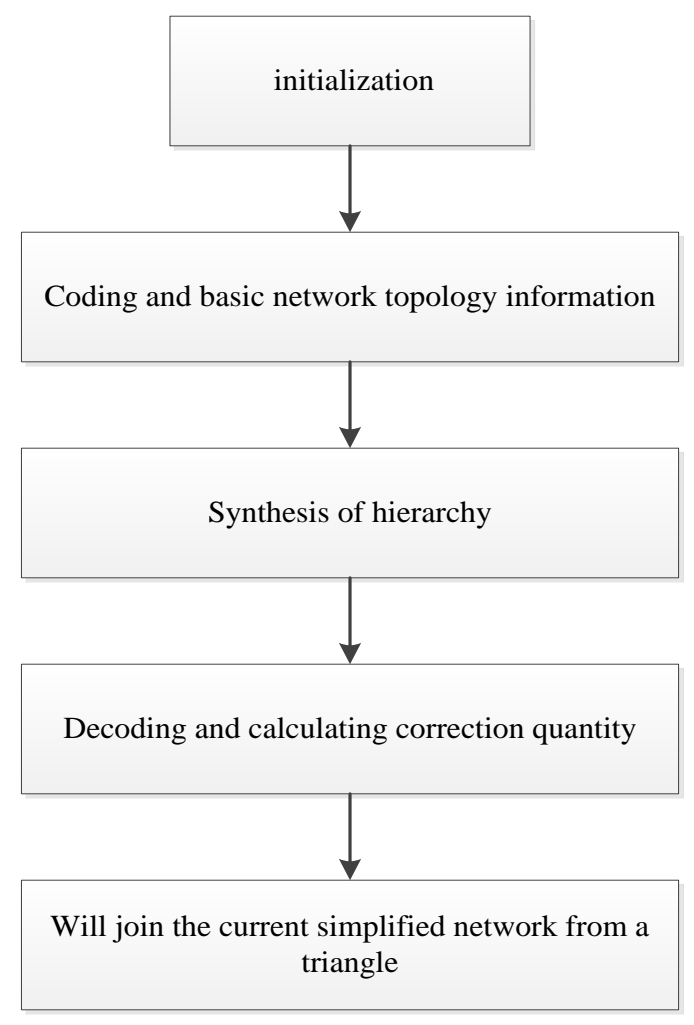

Figure 3. Decoding Description 


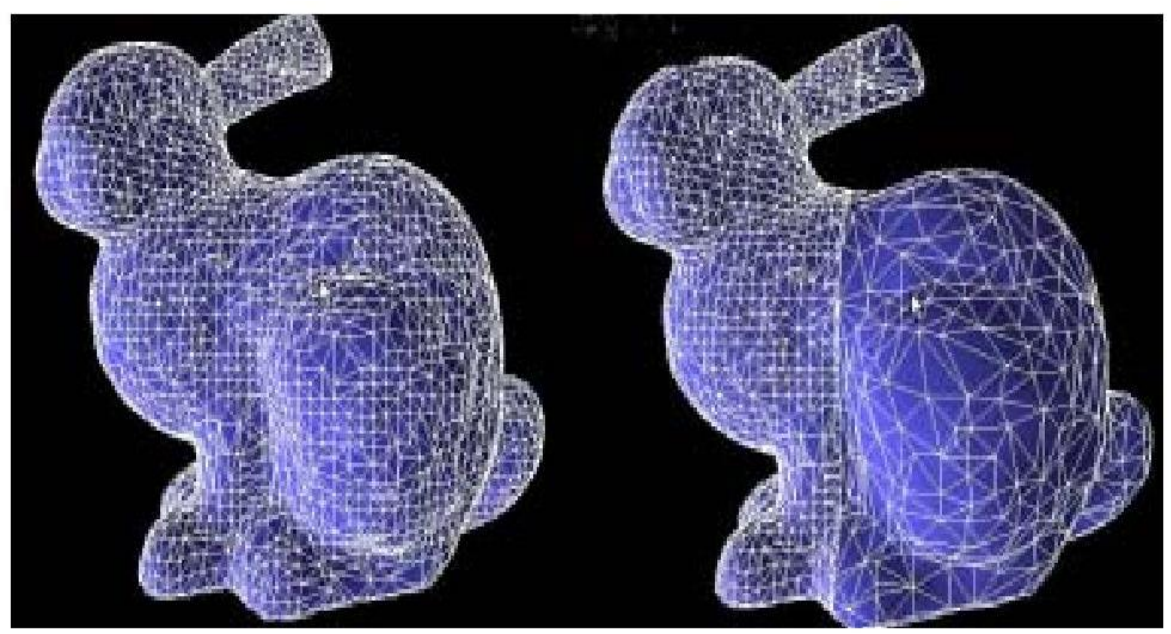

Figure 4. Bunny Model

\section{The Test and its Result}

In order to compare the efficiency with other modeling algorithm, this test is run on the common Bunny model. By setting up the Bunny's BSP two branch tree at the end of receiving portal, the relevant layer of the Bunny's frontal contour and the information of the visible triangle can be obtained, as shown in Figure 4. The back of the bunny is sketched through the primitive grid data, doing so will cause the triangle and climax's data transfer flow reduced by $60 \%$ without influencing the receiving end's sensitive data, in terms of data processing and displaying. As it turned out, the mechanism of this algorithm can achieve a result that is of high satisfaction. The flaw, however, is the transposing binderies appears to be imminent, which, however, can be solved by adding a transposing section in between.

\section{Conclusion}

This article is dedicated to the purpose of introducing the simplification of the triangular-network's multi-resolution, as well as the concept of grid, trigonometry, topographic goading, triangular-sector, etc. In addition, this article proposes a new algorithm that combines the layer-segmentation of the multi-resolution model and the BSP tree, in which the layer-segmentation of the triangular belt is capable of achieving a better result in terms of dissolving the model, therefore creating the foundation of the BSP tree's structural setup. The purpose of finding BSP tree, on the other hand, is to identify sensitive data from the non-sensitive one, so that whatever data that is contacted with the viewpoint can be fully transferred and displayed. Speaking of the designing part of the database, this algorithm combines the latest techniques such as the XML.ADO.NET, and thereby capable of designing transfer plans accordingly.

\section{Acknowledgment}

The author is grateful to the referee for his/her helpful comments. This work was supported by Educational Commission of Fujian Province, China (Grant No. JA13235) and Natural Science Foundation of Fujian Province, China (Grant No. 2015J01670).

\section{References}

[1] M. R. Jardin, J. Remy and D. Artaud, "Volume rendering of the tracheobronchial tree: clinical evaluation of bronchographic images", Radiology, vol. 20, no. 8, (1998), pp. 761-70.

[2] S. D. Bergen, C. A. Ulbricht and J. L. Fridley, "The validity of computer-generated graphic images of forest landscape", Journal of Environmental Psychology, vol. 15, no. 1, (1995), pp. 135-146. 
[3] R. Soummer, A. Ferrari and C. Aime, "Speckle noise and dynamic range in coronagraphic images", Astrophysical Journal, vol. 66, no. 9, (2007), pp. 642-656.

[4] S. Seidenari, A. Conti and P. Pepe, "Quantitative description of echographic images of morphea plaques as assessed by computerized image analysis on $20 \mathrm{MHz}$ B-scan recordings", Acta dermatovenereologica, vol. 75, no. 2, (1995), pp. 442-445.

[5] Y. Y. Gleizer, G. A. Zubovskii and A. M. Soskin, "METHODS OF OBTAINING ROENTGENSCANNOGRAPHIC IMAGES", vol. 75, no. 2, (2005), pp. 43-46.

[6] C. A. Sneiderman, J. P. Cookson and A. F. Hood, "Use of computer graphic images in teaching dermatology, Computerized Medical Imaging \& Graphics", Official Journal of the Computerized Medical Imaging Society, vol. 16, no. 22, (1992), pp. 151-152.

[7] S. Azzaretti, D. Dotti and R. Lombardi, "Echo-graphic images enhanced through Walsh functions , Proceedings of the IEEE Ultrasonics Symposium, vol. 1, no. 3, (1997), pp. 675-678.

[8] E. Nakamae, K. Kaneda and K. Harada, "Reliability of computer graphic images for visual assessment", Visual Computer, vol. 7, no. 6, (1991), pp. 138-148.

[9] D. M. Hynes, L. Mazurkewich and J. A. Rowlands, "Preliminary experience with acquisition of 2048fluorographic images", Proceedings of SPIE - The International Society for Optical Engineering, vol. 21, no. 63, (1994), pp. 158-164.

[10] B. C. Gray, S. V. Sohoni and W. M. Strobel, "Method and apparatus for producing signs with prismatic letters and graphic images", vol. 2, no. 6, (2000), pp. 23-26.

[11] A. M. Grishin, "On some relations between graphic images and sounds", Prikl.diskr.mat, vol. 23, no. 2, (2009), pp. 103-105.

[12] R. J. Bill, "Machine for forming improved graphic images on substrates", vol. 23, no. 5, (1999), pp. 5659.

[13] M. I. Devishev and I. V. Koptev, "Computer reconstruction of scientigraphic images", Biomedical Engineering, vol. 12, no. 3, (1978), pp. 298-302.

[14] N. Nishikawa and A. Suzuki, "Computer graphic images of 3-D flows", Journal of the Visualization Society of Japan, vol. 6, no. 5, (1986), pp. 56-59.

[15] A. Boutoub and H. Ettouati, "Numerical simulations of radiative heat transfer in cylindrical participating enclosures using the finite volume method", International Journal of Heat \& Technology, vol. 22, no. 2, (2004), pp. 147-, 2004.

[16] E. A. Chinnov and O. A. Kabov, "Heat transfer and breakdown of subcooled falling liquid film on a vertical middle size", International Journal of Heat \& Technology, vol. 20, no. 2, (2002), pp. 69-78.

[17] M. Quintard and J. R. Puiggali, "Numerical modelling of transport processes during the drying of a granular porous medium", International Journal of Heat \& Technology, vol. 4, no. 2, (1986), pp. 37-57.

[18] J. Chengfeng, W. Zhang and Y. Ying, "A micro-gesture recognition on the mobile web client", Review of Computer Engineering Studies, vol. 2, no. 2, (2015), pp. 19-24.

[19] R. Jin, L. Hong, C. Wang, L. Wu and W. Si, "A Hierarchical clustering community algorithm which missed the signal in the process of transmission”, Review of Computer Engineering Studies, vol. 2, no. 3, (2015), pp. 27-34.

[20] Y. Zhao, C. Feng, J. Yang and L. Wang, "LITERATURE REVIEW OF NETWORK PUBLIC OPINION ABOUT THE E-COMMERCE", Review of Computer Engineering Studies, vol. 2, no. 2, (2015), pp. 25-,2015.

[21] J. H. Tu and Z. Quan, "Design and implementation of the crying voice detection circuit in the baby's supervision system", Review of Computer Engineering Studies, vol. 1, no. 1, (2014), pp. 13-16.

[22] R. Jin, K. Chunhai and R. Liu, "Biclustering algorithm of differential co-expression for gene data , Review of computer engineering studies, vol. 10, no. 102, (2014), pp. 7-12.

[23] W. Ya and S. Qin, “Aircraft fault diagnosis by solving map exactly”, Review of Computer Engineering Studies, vol. 2, no. 1, (2015), pp. 1-8. 